\title{
The Research on Transmission Reliability Algorithm Based on Power Control in the Internet of ships
}

\author{
Wang Yizhen ${ }^{1, a}$, Sun Yongqiang ${ }^{2, b}$, and ${ }^{*}$ Sun $\mathrm{Yi}^{3, \mathrm{c}}$ \\ ${ }^{1,3}$ Beijing University of Posts and Telecommunications, Beijing 100876, China \\ ${ }^{2}$ China Waterborne Transport Research Institute \\ aemail: 2013213437@bupt.edu.cn, bemail: sunyq@wti.ac.cn, 'email: sunyisse@bupt.edu.cn
}

\begin{abstract}
Keywords:The Internet of ships; system architecture; positioning; power control.
Abstract. The Internet of ships is the application of Internet of things in the intelligent transportation system, which has attracted the attention of domestic and international relevant research institutions. This paper introduces the basic concepts of the Internet of ships, the research analyzes some technical problems in the development process of the Internet of ships, mainly about RFID, middleware technology and so on. The location of the ship nodes is the core technology of the the Internet of ships. It is not only related to the safety of the ships, but also affect its the development prospects. In this paper, based on the problem of poor transmission reliability of the communication between a ship and a ship, and based on the distance between the transmitter and the receiver, a minimum maximum power control transmission reliability evaluation (TDOA-SMMPETR) algorithm is proposed. Technology of the Internet of ships involves many disciplinary fields, to be further studied and discussed in the future.
\end{abstract}

\section{Introduction}

The Internet of ships connect vessels together using an Internet, which is a network, based on realizing the goal of shipping and refinement of management, service industry to achieve comprehensive and travel experience of humanity, and it combines with the intelligent shipping information service of IOT technology.

The Internet of ships is putting the concepts and technology of IOT into the field of inland river shipping, which is a new direction and has a certain creative and exploratory. Under the background of the vigorous development of the Internet of things industry, it is based on the current situation and existing problems in the inland areas of information services, combined with various levels of demand of government, industry and the public, determining the position of vessel network, and sorting some priorities of demand. Demand analysis is the basis of all the work. Only based on the demand, can we clear the function, locate the data source, determine the appropriate technology, and then build the system.

The Internet of ships is the application of Internet of things technology in the intelligent transportation system, which has attracted the high attention of domestic and international relevant research institutions. This paper introduces the basic concept of the ship, the study analyses some technical problems of the development process of the Internet of ships, mainly about RFID, middleware technology and so on.

The positioning of the ship node is the core technology of the Internet of ships, which is not only related to the safety of the ship, but also affect the development prospects of the Internet of ships.

In this paper, a minimum maximum power control transmission reliability evaluation algorithm TDOA-SMMPETR is proposed, which is based on the problem of poor transmission reliability of the communication between the ship and the ship, and based on the distance between the transmitter and the receiver.

\section{Key technologies of the Internet of ships}

There are still some technical problems to be solved in the popularization and development of the Internet of ships. These problems are related to each layer of the Internet of ships.

$\square$ RFID 
RFID(radio frequency identification)is a non-contact automatic identification technology, and it automatically identifies the target object and obtains the relevant data through radio frequency signals, recognition work without manual intervention. At present, RFID technology has been applied in many fields, such as logistics and supply chain management, no parking charge system, and so on.

The main advantage of RFID is that the technology can identify many high-speed moving objects and can easily realize the data transmission between nodes in the Internet of ships. In the Internet of ships, it is recommended to use active RFID technology to achieve communication. Active RFID can provide further reading and writing distance, and can realize the active perception, which is important to the Internet of ships. Compared with the other transmission technologies, RFID also has the advantages of large amount of data storage, compact and lightweight, long service life for using, waterproof, antimagnetic, good safety and so on.

Using Ship automatic identification system and the installation of RFID devices on the ship lock and ship, realize automatic identification of the ship's identity and judgment of the running state, and provide support for boater handling the formalities of lockage, lock management department organization and management of ship lockage.

$\square$ Middleware Technology

Middleware Technology is the core technology in the field of software development. In the field of Internet of things, we also need to focus on the research and development of RFID middleware in the Internet of things.

RFID middleware is an intermediate program realizing data transmission, filter, data format conversion between RFID hardware devices and the application system. It makes the data and information read and write by RFID reader extract, decrypt, filter, format conversion, import into the application program of the Internet of ships by middleware and supplies for users to use by system application reacting on the user interface.

According to the different application of the Internet of ships, we can develop different RFID middleware.

$\square$ Protocol development

The Internet of ships don't use the traditional TCP / IP reference model, and develop a new network protocol stack which is suitable for. The research and development of the new protocol should also refer to the idea of OSI network layer, and discuss the system structure of the Internet of ships layer by layer. The research and development of the new protocol should introduce the latest research results, and at the same time, combined with the actual characteristics of the Internet of ships and should pay attention to the operational efficiency of the protocol.

The Internet of ships also need to switch-in the Internet, which requires to research the protocol conversion issues, so that the data of the Internet of ships and data in the Internet achieve interoperability. Of course, the network protocol of the Internet of ships also includes network control, data security transition and other aspects of the network control.

$\square$ Intelligence technology

The Internet of ships is a new type of intelligent network, using some advanced intelligent technology in the Internet of ships. Through the intelligent technology can make a ship intelligent, it can take the initiative to perceive the environment changes and real-time traffic conditions even the driver's demand and so on.

The main contents of intelligent technology research include artificial intelligence theory, intelligent control system, signal processing and identification, information fusion and other aspects of the content. The expected ship driverless, and traffic intelligent navigation applications to use intelligent technology as the foundation.

\section{- Safe reliability}

Building a safe and reliable Internet of things system is a hot and difficult technology. Security and reliability will determine the extent of the popularization of the Internet of ships. Patent, 
inclusiveness and anonymity of the Internet of ships has brought some potential inevitable security risks.

The complex and poor application environment of the Internet of ships requires high reliability. The Internet of ships should have the ability to defending against network attacks, protecting the privacy of individuals, and ensuring the accuracy of data transmission.

\section{Determination of the distance of the nodes in the Internet of ships.}

- Use the time difference of arrival (TDOA). The positioning principle of TDOA is based on the time difference of different signal transmission to calculate the distance between nodes.

In the specific implementation, we usually install signal transmitters at the location source with different transmission rates (such as ultrasonic and radio frequency), the receiver can get the distance between the source and the location source by calculating the time difference of the different kinds of signals.

For example, the location source transmit two kinds of wireless signals at the same time with the transmission rate are $c_{1}$ and $c_{2}$, the receiver node detects and records arriving time pf two kinds of signals are $\tau_{1}$ and $t_{2}$.

Because the ocean will exist some influences of some environmental factors, the most important effect is wind, so we define environmental factor $\varepsilon$, then the distance between two nodes is

$\mathrm{d}=\left[\left(\mathrm{t}_{2}-\mathrm{t}_{1}\right) \times \frac{\mathrm{c}_{1} \mathrm{c}_{2}}{\mathrm{c}_{1}-\mathrm{c}_{2}}\right] \mathrm{c}$

According to the study, the size of the wind is represented as the wind speed, The effect of the wind speed on distance is a function. We assume the relation of them in the Table-wind as follows: Table-wind

\begin{tabular}{|l|l|l|}
\hline Wind force & Wind speed $(\mathrm{m} / \mathrm{s})$ & \multicolumn{1}{|c|}{$\varepsilon$} \\
\hline 0 & 0 & 1 \\
\hline 1 & $1.6-7.9$ & 0.95 \\
\hline 2 & $8.0-13.8$ & 0.90 \\
\hline 3 & $13.9-20.7$ & 0.85 \\
\hline
\end{tabular}

The $\varepsilon$ is 1 when the sea is calm,and when the wind speed increases, the $\varepsilon$ is smaller and the distance between two nodes will reduce.

-The relation between the wireless signal transmitting power $p_{1}$ and receiving power $p_{s}$. with their distance as follows:

$$
\mathrm{p}_{\mathrm{r}}=\frac{\mathrm{p}_{\mathrm{t}}}{\mathrm{d}^{\mathrm{n}}}
$$

Among them, $\mathrm{n}$ is the propagation factor associated with the signal propagation environment, using the logarithm operation, the calculation formula (7) can be got:

$p_{\mathrm{r}}(\mathrm{dBm})=\Lambda-10 \mathrm{n} \log \mathrm{d}$

A represents received signal power of signal transmission distance of $1 \mathrm{~m}, \mathrm{~A}$ and $\mathrm{n}$ are all the values we should calculate according to the specific situation.

\section{Transmission Reliability Algorithm Based on Power Control in the Internet of ships}

Aiming at solving the problem of poor transmission reliability of the communication between the ship and the ship, this paper put forward to a Maximum Power Minimum Transmission Reliability of Evaluation TDOA-SMMPETR (MMPETR) algorithm., based on the corresponding minimum maximum power control Evaluation of Transmission Reliability algorithm and combined with methods of the arrival time difference to determine distance between transmitter and receiver.

To study the influence of transmission power of the mobile ship control technology on the reliability of data transmission, at the same time determining the distance between transmitter and receiver and improvement to the existing ETR algorithm to improve the success rate of message transmission between the communication links. 
Fast moving of the ship will lead to the wireless signal attenuation, can adopt Rayleigh attenuation model. Simulate wireless signal propagation process in the ocean environment. $\mathrm{p}_{\mathrm{r}}$ is a received signal strength of nodes whose distance to wireless signal transmitter is $\mathrm{d}$, which is an exponentially distributed random variables. The probability density function for $p_{r}$ is $f_{\text {rev }}\left(p_{r}\right)$ :

$f_{r \theta v}\left(p_{r}\right)=\frac{1}{E\left(p_{r}\right)} \exp \left[-\frac{p_{r}}{E\left(p_{r}\right)}\right]$

$\mathrm{E}\left(\mathrm{p}_{\mathrm{r}}\right)$ represents the mean of $\mathrm{p}_{\mathrm{r}}$.

According to the document[1], and assuming that the received signal follow the Friis rule, the mean of $p_{r}, E\left(p_{r}\right)$ is:

$$
E\left(p_{r}\right)=\begin{gathered}
{ }_{t} G_{t} G_{2} \lambda_{c}^{2} \\
16 l^{2} d^{2}
\end{gathered}
$$

$$
\lambda_{s=}=\mathrm{c} / \mathrm{I}
$$

Among them, $p_{r}$ represents the value of the wireless transmitting power of the ship transmitter; $i_{t}$ represents the signal transmitting antenna gain; $\left\langle i_{r}\right.$ The signal receiving antenna gain; $\lambda_{c}$ represents radio signal wavelength represents light speed; $f$ represents radio signal frequency; $d$ represents the distance between transmitter and receiver.

According to the definition of the IEEE 802.1 1P protocol standard, if signal strength of the received alarm message by the ship is higher than the minimum signal receiving sensitivity $\mathbf{P}_{\min }$, then can decode the received wireless signal and acquire real contents of the alarm message; otherwise the decoding is failed, and the alarm message is discarded.

Therefore, for the ship receiver with the distance $d$, the probability of alarm messages are successfully transmitted between is defined as the probability of the signal receiving sensitivity of the ship receiver is greater than the minimum signal receiving sensitivity $\mathrm{p}_{\min }$. That probability is $p_{\text {succ }}(\mathrm{d})$, which at the same time is defined as the message transmission reliability standards. When the distance $d_{1 r}$. between the transmitting warning message ship and ship receiver is $d$ :

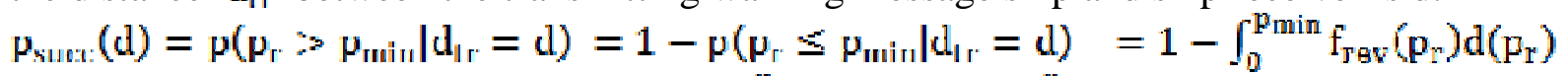

$$
\begin{aligned}
& =\exp \left(-\frac{p_{m i n}}{F(\mu \mathrm{r})}\right)=\exp \left(-\frac{p_{\min }}{\mathrm{E}\left(\frac{p_{\mathrm{t}}}{\mathrm{d}^{\mathrm{L}}}\right)}\right)
\end{aligned}
$$

Put (2)into (4):

$$
\mathrm{p}_{\text {succ }}(\mathrm{d})=\exp \left(-\frac{1 \epsilon \pi^{2} \mathrm{~d}^{2} \mathrm{p}_{\min }}{\mathrm{p}_{\mathrm{t}} \mathrm{G}_{1} \mathrm{G}_{\mathrm{r}} \mathrm{h}_{\mathrm{c}}^{2}}\right)=\exp \left(\mathrm{K} \cdot \mathrm{C} \cdot \mathrm{d}^{2}\right)=\exp \left(\mathrm{K} \cdot \mathrm{C} \cdot\left[\left(\mathrm{t}_{2}-\mathrm{t}_{1}\right) \times \frac{\mathrm{c}_{1} \mathrm{c}_{2}}{\mathrm{c}_{1}-\mathrm{c}_{2}}\right]^{2} \varepsilon^{2}\right)
$$

Among, $\mathrm{K}=-\frac{16 \pi^{2}}{\lambda_{\mathrm{c}}^{2}} ; \mathrm{C}:=\frac{\mathrm{l} \mathrm{min}}{\mathrm{p}_{\mathrm{t}}}$

$\mathrm{P}_{\mathrm{Th}}$ is defined as the threshold of the reliable transmission of the alarm message, which as a system parameter in the Internet of ships, the value can be set flexibly according to the demand of the system. In order to ensure the reliability of alarm message transmission network in the Internet of ships, so the successful hop transmission probability of any two ships whose distance is d can not be lower than the threshold. That is:

$p_{\text {succ }}(d)>p_{\text {Th }}$

So, when the alarm messages are received successfully with probability $\mu_{\text {Th }}$, transmitting power transmitter ship required is $\mathrm{p}_{\mathrm{t}, \mathrm{Th}}$, which satisfies (7):

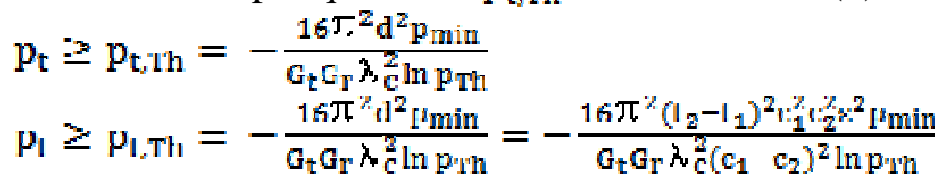

The document [1] proposes a reliable multi-hop broadcast protocol based on delay perception (Delay - aware Reliable Broadcast Protocol, DRBP). Assume that in the traffic scene, the ship with the number $s_{i}$ need to send a warning message to the rear danger zone, record all the neighbors ships are $\left\{B_{i}\right\}$,all ships in $\left\{B_{j}\right\}$ consisting of the number of communication links with $s_{i}$ is $\left|B_{j}\right|$. 
Work process of DRBP protocol are as follows:

Step 1 Calculate the transmission reliability $p_{\text {succ }}$ (d)of $\left|\|_{1}\right|$ links.

Step 2 Calculate the index $\mathrm{D}^{*}$ of real-time transmission of $\left|H_{1}\right|$ links

Step3 Generate comprehensive evaluation of link quality index $E^{*}$ of $\left|B_{i}\right|$ links

According to the relationship of specific value between $\mathrm{E}^{*}$ and $\mathrm{E}_{\mathrm{Th}}^{*}$ choose optimal relay. $\mathrm{E}_{\mathrm{Th}}^{*}$ is the lowest threshold for the index of comprehensive evaluation of the DRBP protocol in the link quality. This shows that the calculation of the transmission reliability is the precondition for choosing the optimal relay. In this paper the $s_{\mathbf{k}}$ indicates ship in the best optimal relay candidate set $\left\{B_{1}\right\}$, that is $s_{k} \in\left\{B_{1} \mid\left(k=1,2, \ldots\left|B_{i}\right|\right)\right\}$.

Calculate the transmission reliability $\mu_{\mathrm{sus}: \mathrm{s}}\left(\mathrm{d}_{\mathrm{i}, \mathrm{k}}\right)$ between the transmitter $s_{\mathrm{j}}$ and the receiver $s_{\mathrm{k}}$. $d_{i, k}$ represents the distance between ship transmitter and receiver.

\section{Algorithm TDOA-SMMPETR}

For ship $s_{k}, k=1$ to $s_{i}$

1) Computation of distance

between and

$=$ , install signal transmitters at , which is as the location source.

2)Computation the need transmission power $p_{t}$ of $s_{i}$

$\mathrm{P}_{\mathrm{t}}=-\frac{16 \pi^{2} \mathrm{l}_{\mathrm{i}, \mathrm{I}}^{\mathrm{I}} \mathrm{min}}{\mathrm{G}_{\mathrm{t}} \mathrm{G}_{\mathrm{r}} \boldsymbol{h}_{\mathrm{g}}^{2} \ln \mathrm{p}_{\mathrm{Th}}}$

3)If $p_{i, \text { min }}: p_{t}$, then $p_{\text {suce }}\left(d_{i_{k} k}\right)=p_{T h}$

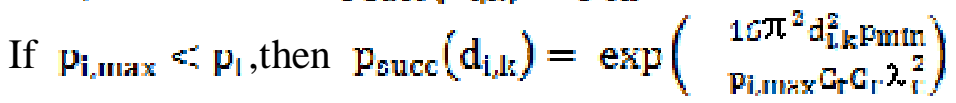

Else if $p_{i_{\text {m }} \min }<p_{1}<\mu_{i_{\text {n }} \text { max }}$,

then $p_{\text {sucd }}\left(d_{i_{\ell} k}\right)=\theta p_{T h}+(1-\theta) p_{s u c c}\left(d_{i m a x, k}\right) ; \theta=2^{1-\frac{p_{t}}{z}}$

End If

End For

Compare $\mathrm{p}_{\mathbf{t}}$ with the minimum transmission power $\boldsymbol{p}_{\mathrm{i}_{\text {, nin }}}$ and the maximum transmission power $p_{i, \text { max }}$.

-If $\mu_{i_{\ell}, n_{1}} \geq p_{1}$, then think $s_{i}$ has the ability to make a warning message be received by candidate relay with a probability not less than $\mathrm{p}_{\mathrm{Th}}$.

-If $\mu_{\mathrm{i} \text {, nax }} \leqslant \mathrm{p}_{1}$, then think $s_{\mathrm{i}}$ don't have the ability to make a warning message be received by candidate relay with a probability not less than $p_{T h}$. At this time, the value of $p_{s u c c}\left(d_{i_{k} k}\right)$ calculated by the formula (5).Transmitting power of $s_{i}$ is set as $p_{i, m a x}$, the result of the calculation is $p_{\text {succ }}\left(d_{\text {imax,k }}\right)$.

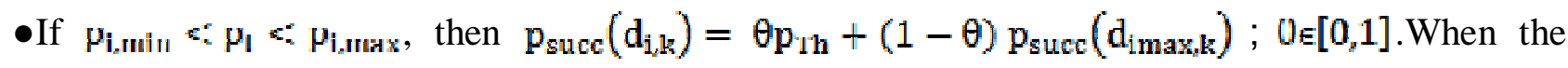
$p_{\mathrm{t}}$ increases, the weight of $\mathrm{p}_{\mathrm{t}}$ reduces, that is the value of $\theta$ is smaller.

\section{Experiment test}

The simulation and experimental values of parameter symbols are shown in table 1 . In order to simplify the model, assuming all ships loaded with the exactly the same wireless communication unit performance. That is to say each ship on the wireless signal transmitting antenna and receiving antenna gain is the same, that is $\mathrm{i}_{\mathrm{t}}=\mathrm{i}_{\mathrm{r}}=1$.

The simulation frequency of wireless signal choose average size of $5.885 \mathrm{GHz}$ and $5.895 \mathrm{GHz}$, that is $5.809 \mathrm{GHz}$. At the same time, through formula (5) draw the change curve of linked transmission reliability of communication with the ship distance $d$ and transmitting power $\mathrm{n}_{\mathrm{t}}$, 
according to the curve we can know that only when $p_{1}=5 \mathrm{dBm}$, $\mathrm{p}_{\text {sum }}$.(d) will show the tendency bigger than 0 ,so select $p_{i, \min }=\mathbf{5} \mathrm{dBm}$. At the same time, when $\boldsymbol{p}_{1}$ increase to $32 \mathrm{dBm}, \boldsymbol{p}_{\text {sum }}$ (d) has already nearly to be 1 , so select $p_{i, \max }=32 \mathrm{dBm}$.

\begin{tabular}{|c|c|c|}
\hline Signal & Description of Parameter & value \\
\hline$\overline{G_{\mathrm{t}}}$ & The signal transmitting antenna gain & 1. \\
\hline $\mathrm{G}_{\mathrm{t}}$ & The signal receiving antenna gain & 1. \\
\hline$\lambda_{c}$ & Radio signal wavelength & $0.0509 \mathrm{~s}$ \\
\hline c & Light speed & 3. $0 \times 10^{a} \mathrm{~m}$ \\
\hline f & Radio signal frequency & 5. $809 \mathrm{GHz}$ \\
\hline $\mathrm{p}_{\min }$ & Xinimum sensitivity of received signal & $-87 \mathrm{dBa}$ \\
\hline$p_{i \text { min }}$ & Minimu transmit power of ship wireless coenunication & $5 \mathrm{dBa}$ \\
\hline$p_{\text {imax }}$ & Yaximum transmit poser of ship wireless communication & $32 \mathrm{dBd}$ \\
\hline $\mathrm{p}_{\mathrm{Th}}$ & The transmission success rate threshold of alarm message & 0.96 \\
\hline s & Risk area for warning messages & $200=$ \\
\hline
\end{tabular}

Test the relationship of the wind speed and the environmental factor in Figure01.We can verify the table-wind.
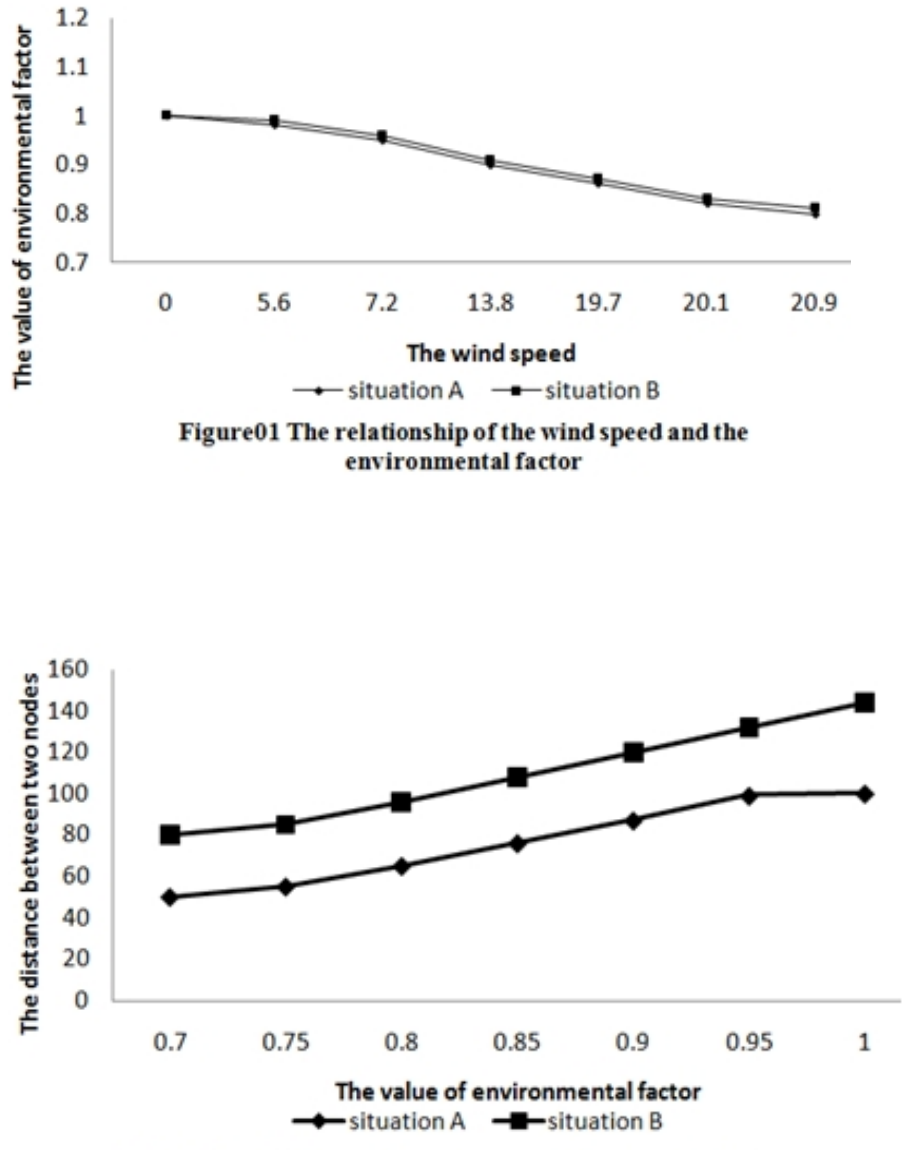

Figure 0 The influnce of environmental factor on the distance

In Figure0, it shows the influence of the environmental factor on the distance.

Figure1 shows the effect of distance between transmit and receive ship on transmission success rate. 


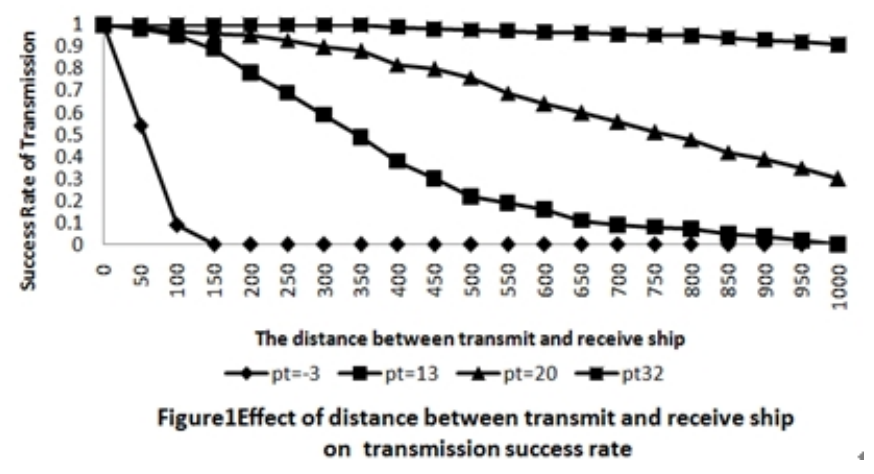

Figure1 represents that the success rate of message transmission reliability reduces when the distance $d$ increases, especially when the transmit power $=3 \mathrm{dBm}$, link reliability decreased sharply with the increase of $d$.

\section{Conclusions}

According to the results of the experiment test, the environmental factor has influence of the distance. The algorithm takes into account all situations of the relationship between the transmitting power of ship transmitter required successfully distributed alarm message and the minimum and maximum transmit power, and analyzes the impact of the mobile ship power communication link control technology on data transmission reliability; and according to the dynamic curve of message transmission success rate with the change of the transmitting power to determine the specific number of ship transmitter $s_{k}$ the minimum and maximum transmission power. And we should do more research on the stability of the TDOA-SMMPETR algorithm.

\section{Acknowledgements}

This work is a basic research project of China Waterborne Transportation Institute prospective.

\section{References}

[1] GUO W. Research on message transmission of safety-related applications in VANET[D].Hefei: University of Science and Technology of China,2014

[2] Rong na $\mathrm{Hu}$, Aihuang GUO. Transmission reliability algorithm based on power control in Internet of vehicles. The number of clarification is TP393 . 03 The number of document is A.

[3] Jianqiang Wang,Chenwen Wu,Xiaojun Li. Research on Architecture and Key Technologies of Internet of Vehicles.The passage number is : 1008--0570(2011)04-0156-03. The number of clarification is TP393 The number of document is A.

[4] Qun Wang, Huanyan Qian, Liang Zhang. Localization and Nodes Location-Aware in Internet of Vehicles. The passage number is 1001-4616(2015)01-0066-09 ] The number of clarification is TP393.03 The number of document is A.

[5] Wenming Qin,Xiaofeng Wang. A Survey on Netting with Internet of Vessels.The passage number is : 1000-4653(2015)02-0001-04. The number of clarification is TP393 he number of document is $\mathrm{A}$. 